Article

\title{
Elevated brachial-ankle pulse wave velocity is associated with an increase of left ventricular mass index in hypertensive patients
}

\author{
Dian Fajarwati' ${ }^{1}$ Budi S Pikir1, Rizal Muhammad1', Eka Prasetya Budi Mulia1*
}

\author{
${ }^{1}$ Department of Cardiology and Vascular Medicine, Faculty of Medicine, Universitas Airlangga - Dr. Soetomo \\ General Hospital, Surabaya, Indonesia \\ * Correspondence: eka.prasetya.budi-2017@fk.unair.ac.id; Tel.: +62 856-2765-365
}

\begin{abstract}
Background: Brachial-ankle pulse wave velocity (baPWV) is widely used as a marker of arterial stiffness. It has been suggested as a simple method for detecting arterial loads that can trigger left ventricular hypertrophy in the heart. Increased left ventricular hypertrophy in hypertensive patients is a predictor that will have an impact on increasing morbidity and mortality. Objective: This study aimed to evaluate the correlation between the baPWV and the left ventricular mass index (LVMI) in hypertensive patients. Methods: This cross-sectional study was conducted by collecting secondary data from hypertensive patients at the Heart Outpatient Clinic, with inclusion: aged more than 40 years old, suffering hypertension more than one year, and had baPWV and echocardiography data. Correlation between baPWV and LVMI was evaluated using the Pearson correlation test. Results: A total of 40 hypertensive patients were enrolled in this study. $60 \%$ were women. The mean age was $52.92 \pm 9.50$ years. Mean LVMI was $107.20+21.01 \mathrm{gram} / \mathrm{m} 2$. LVMI showed a significant association with age and systolic blood pressure $(\mathrm{p}=0.001$ and 0.019 , respectively). Mean baPWV was $1832.90+336.22 \mathrm{~cm} / \mathrm{sec}$. A significant association was found between baPWV and age and systolic blood pressure ( $\mathrm{p}=0.000$ and 0.049 , respectively). There was a significant positive correlation between baPWV and LVMI ( $\mathrm{r}=0.493, \mathrm{p}=0.001)$; and between baPWV and Relative Wall Thickness (RWT) $(\mathrm{r}=0.404, \mathrm{p}=0.01)$. Conclusion: This study shows that elevated baPWV is an indicator of increased LVMI in hypertensive patients. Examination of baPWV should be carried out routinely to detect earlier arterial stiffness as a morbidity and mortality prevention strategy in hypertensive patients.
\end{abstract}

Keywords: arterial stiffness; brachial-ankle pulse wave velocity; left ventricular mass index; echocardiography; hypertension

\section{Introduction}

Hypertension is the leading cause of cardiovascular morbidity and mortality. More than a quarter of the adult population in the world suffers from hypertension; about 1 billion people in 2000 and will increase by $29 \%$ or around 1.56 billion people in 2025.[1] In Indonesia, based on the results of 2007 basic health research, the prevalence of hypertension is $29.8 \%$.[2]

Increased afterload in hypertension causes an increase in left ventricular wall thickening. This situation increases the risk of cardiovascular disease earlier. Epidemiological studies showed left ventricular hypertrophy is associated with increased morbidity and mortality, including the incidence of coronary heart disease and cardiovascular death.[3]

Recently, more attention is given to the relationship between arterial stiffness and cardiovascular disease. Arterial stiffness is mainly involved in the development of isolated systolic hypertension, especially in the elderly population and is associated with increased mortality and morbidity. Pulse pressure and pulse wave velocity (PWV) represent measurements of arterial stiffness, indicating that arterial stiffness increases with age and certain disease conditions associated with increased cardiovascular risk, including hypertension, diabetes mellitus, and hypercholesterolemia.[4] 
Changes in arterial stiffness can be detected earlier before clinical symptoms arise and act as a marker of the development of atherosclerosis in the future. Therefore, doctors need automatic devices that do not require special skills operators to evaluate arterial stiffness. Echocardiography is currently widely used to determine the presence of left ventricular hypertrophy by measuring the left ventricular mass index. However, it needs to be carried out by a special operator and serves as a means of evaluating because it is ideally carried out serially and is more sensitive than electrocardiographic examination.[5] Pulse wave velocity (PWV) is an agreed-upon method used to measure arterial stiffness noninvasively. It is currently considered the golden standard index that provides strong mortality and cardiovascular events predictions to evaluate arterial flexibility between two points in arterial branching. PWV uses automatic devices that are easy to operate and save more time because no special skills are needed to use the probe.[6] It is important to not only reduce blood pressure but also reduce arterial stiffness to improve prognosis in patients with hypertension. This study aims to determine the correlation between the brachial-ankle pulse wave velocity and the left ventricular mass index in hypertensive patients.

\section{Materials and Methods}

This study was a cross-sectional analytic observational. The Hospital Ethical Committee approved the study (0710/2012). Patients who took part in the study were patients with a history of hypertension more than or equal to one year, aged $>40$ years, and patients who had data on examination of the brachial-ankle pulse wave velocity and echocardiography. Patients with a history of coronary heart disease, diagnosed with peripheral artery disease, a history of cerebrovascular disease, a history of chronic kidney disease, anemia, and a history of hyperthyroidism were excluded.

Medical records of patients who came to the cardiac clinic were collected, then the medical record selection was conducted, and those who met the inclusion criteria were included as samples in the study. The data recording includes the identity of the subject, the results of an examination of the brachial-ankle pulse wave velocity, and the results of the echocardiography examination. The brachial-ankle pulse wave velocity was measured using OMRON VP-2000/1000 type 230 Non-invasive vascular screening device BP- 203RPE II (Omron Healthcare, Kyoto, Japan). Siemens ACUSON X500 Ultrasound Imaging System (Siemens Healthcare $\mathrm{GmbH}$, Erlangen, Germany) was used for echocardiography examination. The data collected will be processed, analyzed, and subsequently reported The data will be analyzed analytically using the Spearman correlation test (if the data is abnormally distributed) or the Pearson correlation test (if the data is normally distributed). Data analysis results will be displayed in graphical form. The entire data analysis process will be processed using the SPSS version 20 computer program.

\section{Results}

\section{Characteristics of subjects}

Hypertension was experienced by 16 men and 20 women (table 1). The mean age was $52.92 \pm 9.50$ years. Most of the patients aged 40-49 years were $45 \%$, followed by $50-59$ for $40 \%$. The mean systolic blood pressure is $151.47 \pm 17.55 \mathrm{mmHg}$, and the mean diastolic is $86.2 \pm 11.55 \mathrm{mmHg}$. The patient's body mass index in this study had a mean of $26.12 \pm 4.73$. Subjects with hypertension who were also obese were seven patients (17.5\%), 14 patients with smoking (35\%), 30 patients with dyslipidemia (75\%), 13 patients with hyperuricemia $(32.5 \%)$, and 13 people with diabetes mellitus (32, 5\%). In comparison, the antihypertensive drugs obtained by hypertensive patients in this study were 25 Calcium Channel Blockers (62.5\%), 23 ACE Inhibitors (57.5\%), 16 patients with Angiotensin Receptor Blocker (40\%), $\beta$ blockers 19 patients (47.5\%), and seven patients with diuretics $(17.5 \%)$.

Table 1. Characteristics of subjects

\begin{tabular}{llc}
\hline \multicolumn{1}{c}{ Characteristics } & \multicolumn{1}{c}{ Statistic/ Category } & Results \\
\hline Age (year) & Mean $\pm \mathrm{SD}^{\mathrm{a}}$ & $52,92 \pm 9,50$ \\
& Range & $40-82$ \\
Age (category) & $40-49$ year & $18(45 \%)$ \\
& $50-59$ year & $16(40 \%)$
\end{tabular}




\section{Gender}

Systolic blood pressure

Diastolic blood pressure

Heart rate

Body Mass Index

Obesity

Smoking

Dyslipidemia

Hyperuricemia

Diabetes Mellitus

Antihypertensive therapy

Calcium Channel Blocker

ACE inhibitor

Angiotensin Receptor Blocker

$\beta$ - blocker

Diuretic

a SD: standard deviation
$6(15 \%)$

$16(40 \%)$

$24(60 \%)$

$151.47 \pm 17.55$

$119-188$

$86.2 \pm 11,55$

$65-122$

$82.93+17.34$

57-129

$26.12 \pm 4.73$

$19.5-38.9$

$7(17.5 \%)$

$33(82.5 \%)$

$14(35 \%)$

$26(65 \%)$

$30(75 \%)$

$10(25 \%)$

$13(32.5 \%)$

$27(67.5 \%)$

$13(32.5 \%)$

$27(67.5 \%)$

$25(62.5 \%)$

$23(57.5 \%)$

$16(40 \%)$

$19(47.5 \%)$

$7(17.5 \%)$

\section{Characteristics of echocardiography}

Echocardiographic data of patients with hypertension, as shown in table 2, obtained a mean ejection fraction of $69.75+6.89 \%$. The mean cardiac output is $4.83+1.38 \mathrm{~L} /$ minute and cardiac index is $2.86+0.67$. In patients with hypertension, normal left ventricular geometry was eight people $(20 \%)$, concentric remodeling of 10 people $(25 \%)$, concentric hypertrophy of 21 people $(52.5 \%)$, and eccentric hypertrophy in 1 person $(2.5 \%)$.

Table 2. Characteristics of echocardiography

\begin{tabular}{lc}
\hline \multicolumn{1}{c}{ Echocardiography Data } & Mean \pm SD $^{\mathbf{a}}$ \\
\hline EF by Teich (\%) & $69.75 \pm 6.89$ \\
Cardiac Output (L/min) & $4.83 \pm 1.38$ \\
Cardiac Index & $2.86 \pm 0.67$ \\
LVIDd (mm) & $44.01 \pm 4.89$ \\
LVIDs (mm) & $26.76 \pm 4.06$ \\
IVST (mm) & $10.73 \pm 1.85$ \\
PWT (mm) & $11.93 \pm 1.90$ \\
RWT & $0.527 \pm 0.10$ \\
LA (mm) & $29.24 \pm 5.12$ \\
LVM (gram) & $209.01 \pm 355.85$ \\
LVMI (gram/ m $\left.{ }^{2}\right)$ & $107.20 \pm 21.01$ \\
E/A & $0.99 \pm 0.30$ \\
Left Ventricle Geometry & \\
Normal & $8(20 \%)$ \\
Concentric Remodeling & $10(25 \%)$ \\
Concentric Hypertrophy & $21(52.5 \%)$ \\
Eccentric Hypertrophy & $1(2.5 \%)$
\end{tabular}




\section{Left Ventricular Mass Index}

Subjects with hypertension obtained a left ventricular mass index (LVMI) between $69.90-149.80$ gram / $\mathrm{m} 2$ with a mean of $107.20+21.01$ gram / m2. The LVMI had a mean of $117.78+22.70 \mathrm{gram} / \mathrm{m} 2$ with a minimum value of $69.90 \mathrm{gram} / \mathrm{m} 2$ and a maximum of 149.80 grams / $\mathrm{m} 2$ in men. LVMI in women patients has a mean of $100.15+16.18$ gram $/ \mathrm{m} 2$ with a minimum value of 80.30 gram / $\mathrm{m} 2$ and a maximum of 141.50 grams / $\mathrm{m} 2$. The LVMI values of male and female hypertensive patients were found to be statistically significant differences (table 3).

Table 3. Comparison between sexes with the left ventricular mass index value

\begin{tabular}{lcccccc}
\hline & n & Mean & SD & Min. & Max. & \\
\hline Men & 16 & 117.78 & 22.70 & 69.90 & 149.80 & \\
Women & 24 & 100.15 & 16.82 & 80.30 & 141.50 & $\mathrm{t}=2.823$ \\
Total & 40 & 107.20 & 21.01 & 69.90 & 149.80 & $\mathrm{p}=0.008^{\mathrm{a}}$ \\
\hline \multicolumn{7}{l}{ a significant at $\alpha=0.05$ (based on Independent t-test) } \\
\hline
\end{tabular}

There was a relationship between LVMI and patient characteristics, where LVMI showed a statistically significant association with age and systolic blood pressure (table 4). There was no significant difference in LVMI in patients who smoked, suffered from diabetes mellitus, dyslipidemia, and hyperuricemia compared to those who did not have these risk factors (table 5).

Table 4. The correlation between LVMI and patient characteristics

\begin{tabular}{lcc}
\hline \multicolumn{1}{c}{ Characteristics } & $\mathbf{r}$ & $\mathbf{P}$ \\
\hline Age & 0.518 & $0.001^{\mathrm{a}}$ \\
Systolic Blood Pressure & 0.370 & $0.019^{\mathrm{a}}$ \\
Diastolic Blood Pressure & 0.127 & 0.435 \\
Heart Rate & 0.040 & 0.806 \\
Body Mass Index & 0.131 & 0.420 \\
\hline
\end{tabular}

a significant at $\alpha=0.05$ (based on correlation test)

Table 5. Comparison of characteristics of patients and LVMI

\begin{tabular}{lcccc}
\hline \multirow{2}{*}{ Characteristics } & \multirow{2}{*}{ Category } & \multicolumn{2}{c}{ LVMI } & \multirow{2}{*}{ Comparison test } \\
\cline { 3 - 4 } & & Mean & SD & \\
\hline DM & Yes & 116.42 & 16.98 & $\mathrm{t}=-1.997$ \\
Smoking & No & 102.77 & 21.59 & $\mathrm{p}=0.053$ \\
& Yes & 115.48 & 22.58 & $\mathrm{t}=-1.887$ \\
Dyslipidemia & No & 102.75 & 19.09 & $\mathrm{p}=0.067$ \\
& Yes & 107.07 & 22.55 & $\mathrm{t}=0.054$ \\
Hyperuricemia & No & 107.52 & 16.55 & $\mathrm{p}=0.957$ \\
& Yes & 115.83 & 23.53 & $\mathrm{t}=-1.858$ \\
& No & 103.05 & 18.75 & $\mathrm{p}=0.071$ \\
\hline
\end{tabular}

Brachial-Ankle Pulse Wave Velocity

Brachial-Ankle Pulse Wave Velocity (BaPWV) was obtained between $1139-2675 \mathrm{~cm} / \mathrm{sec}$ with an average of $1832.90 \pm 336.22 \mathrm{~cm} / \mathrm{sec}$. The average BaPWV in male patients was 1865.63 $\pm 322.29 \mathrm{~cm} / \mathrm{sec}$ with the highest value of $2675 \mathrm{~cm} /$ second and the lowest of $1421 \mathrm{~cm} / \mathrm{sec}$. While BaPWV in women with hypertension obtained a mean of $1811.08 \pm 350.28 \mathrm{~cm} / \mathrm{sec}$ with the lowest value of $1139 \mathrm{~cm} / \mathrm{sec}$ and the highest of $2571 \mathrm{~cm} / \mathrm{sec}$. There were no statistically significant differences in BaPWV values between men and women (table 6).

Table 6. Comparison between sexes towards the value of BaPWV

\begin{tabular}{lcccccc}
\hline & N & Mean & SD & Min. & Max. & \\
\hline Men & 16 & 1865,63 & 322,29 & 1421 & 2675 & \\
\hline Women & 24 & 1811,08 & 350,28 & 1139 & 2571 & $\mathrm{t}=0,498$ \\
\hline Total & 40 & 1832,90 & 336,22 & 1139 & 2675 & $\mathrm{p}=0,622$ \\
\hline
\end{tabular}


This study showed a statistically significant association between BaPWV and the subject's age and systolic blood pressure, as shown in table 7 and table 8 .

Table 7. BaPWV correlation with patient characteristics

\begin{tabular}{lcc}
\hline \multicolumn{1}{c}{ Characteristics } & $\mathbf{r}$ & $\mathbf{P}$ \\
\hline Age & 0.658 & $0.000^{\mathrm{a}}$ \\
Systolic Blood Pressure & 0.314 & $0.049^{\mathrm{a}}$ \\
Diastolic Blood Pressure & 0.140 & 0.390 \\
Heart Rate & 0.006 & 0.972 \\
Body Mass Index & -0.306 & 0.055 \\
\hline a significant at $\alpha=0.05$ (based on correlation test) &
\end{tabular}

a significant at $\alpha=0.05$ (based on correlation test)

Table 8. Comparison of characteristics of patients with BaPWV

\begin{tabular}{llccc}
\hline \multirow{2}{*}{ Characteristics } & \multirow{2}{*}{ Category } & \multicolumn{2}{c}{ baPWV } & \multirow{2}{*}{ Comparison test } \\
\cline { 3 - 4 } & & Mean & SD & \\
\hline DM & Yes & 1961.15 & 324.69 & $\mathrm{t}=-1.715$ \\
\multirow{2}{*}{ Smoking } & No & 1771.15 & 329.73 & $\mathrm{p}=0.094$ \\
& Yes & 1836.21 & 323.92 & $\mathrm{t}=-0.045$ \\
Dyslipidemia & No & 1831.12 & 348.96 & $\mathrm{p}=0.964$ \\
& Yes & 1776.10 & 269.76 & $\mathrm{t}=1.477$ \\
Hyperuricemia & No & 2003.30 & 460.81 & $\mathrm{p}=0.167$ \\
& Yes & 1865.08 & 355.26 & $\mathrm{t}=-0.416$ \\
& No & 1817.41 & 332.48 & $\mathrm{p}=0.680$ \\
\hline
\end{tabular}

Correlation between Brachial-Ankle Pulse Wave Velocity and Left Ventricular Mass Index

The results of the Pearson correlation analysis showed a statistically significant correlation between BaPWV and Relative Wall Thickness (RWT) and BaPWV with the left ventricular mass index. The correlation between BaPWV and RWT is $r=0.404$ and $p=0.01$. In addition, the correlation between $\mathrm{BaPWV}$ and the left ventricular mass index was $\mathrm{r}=0.493$ and $\mathrm{p}=0.001$ showed a moderate positive correlation (table 9).

Table 9. The correlation between the brachial-ankle Pulse Wave Velocity and the Left Ventricular Mass Index

\begin{tabular}{lcc}
\hline \multicolumn{1}{c}{ Correlation } & Correlation coefficient (r) & p \\
\hline BaPWV - RWT & 0.404 & $0,01^{\mathrm{a}}$ \\
BaPWV - LVMI & 0.493 & $0,001^{\mathrm{a}}$ \\
\hline
\end{tabular}

a significant at $\alpha=0.05$ (based on the Pearson correlation test)

\section{Discussion}

Hypertension is a significant cause of cardiovascular morbidity and mortality, affecting almost a quarter of the adult population worldwide. It triggers the underlying cause of left ventricular hypertrophy, determined by several important factors, including volume load, inotropic, and arterial flexibility. The role of arterial stiffness becomes crucial in patients with hypertension. Several methods have been developed to detect and measure arterial stiffness, one of which is a Pulse Wave Velocity (PWV) examination. PWV examination has been recommended by the European Society of Cardiology (ESC) to detect target asymptomatic organ damage in hypertensive patients.

There was a significant correlation between left ventricular mass index and age and systolic blood pressure. Hypertrophy occurs because of the cardiomyocyte response to excessive hemodynamic load, which continues to become myocardial remodeling. Cardiomyocyte hypertrophy causes thickening of the ventricular wall and increases the left ventricular mass is the primary mechanism to reduce stress on the left ventricular wall due to continuous pressure loads.[7] The results of this study are in line with research conducted by Rosendorff et al., who studied 179 men over 75 years of age with a mean age of $81.8 \pm 4.3$ years, averaging $136.5 \pm 18.3 \mathrm{mmHg}$ systolic blood pressure and a mean left ventricular mass index of $136.5 \pm 39.2 \mathrm{~g} / \mathrm{m} 2$. The results showed a significant correlation between systolic 
and LVMI blood pressure with $\mathrm{r}=0.246$ and $\mathrm{p}=0.001 .[8]$

In this study, there was a significant correlation between brachial-ankle PWV and age and systolic blood pressure in hypertensive patients. The prevalence of hypertension increases with age due to changes in arterial structure and function along with the aging process. Large blood vessels become stiffer, which causes blood pressure to increase. Decreased arterial distensibility can be detected in the presence of clinical manifestations in the form of increased pulse pressure and isolated systolic hypertension. $[9,10]$ In the study of the relationship between indicators of arterial stiffness and left ventricular stiffness performed by Minesh et al., 78 people with hypertension and normotension aged 18-91 years showed a strong correlation between femoral heart PWV and age with $r=0.72$ and $p<0.001$. Between the variables, systolic blood pressure and femoral heart PWV also showed a strong positive correlation. The conclusion drawn from the study is that there is a strong correlation between age, systolic blood pressure, arterial stiffness, and left ventricular stiffness.[11]

From the results of the Pearson correlation test that assessed the relationship between brachial-ankle PWV and Relative Wall Thickness (RWT), there was a positive correlation with $r=0.404$ and $p=0.01$. This is in accordance with the research conducted by Schillaci et al., who examined 237 patients with untreated hypertension aged 18-88 years. The results showed a positive correlation between aortic PWV and RWT in patients aged 40-59 years and $>60$ years, whereas, in patients with age group $<40$ years, there was no significant relationship. This is caused by the influence of age is minimal on arterial stiffness at the age of $<40$ years. Whereas in middle age and older, an increase in aortic impedance has a large influence on concentric remodeling without a significant increase in left ventricular mass. As age increases, aortic PWV is a major determinant of large arterial stiffness and is strongly associated with left ventricular concentric remodeling.[12]

Brachial-ankle PWV is an alternative examination to determine arterial stiffness quickly and accurately. Left ventricular mass index value was obtained from echocardiographic examination in hypertensive patients. The results of the two types of examinations were analyzed, and the results showed a positive correlation with $r=0.493$ and $p=0.001$. Printzios et al. developed a study on the relationship of arterial stiffness with systolic and diastolic functions of hypertensive patients who had never received antihypertensive therapy. Two hundred respondents participated in the study consisting of $61 \%$ men and $39 \%$ women, mean systolic blood pressure 165.96 \pm 10.41 , mean femoral carotid PWV 7.96 \pm 1.49 , and mean LVMI $98.14 \pm 12,72$. In his study, there was a significant relationship with the results of a positive correlation between femoral carotid PWV and LVMI, a negative correlation between femoral carotid PWV and ejection fraction, and a negative correlation between femoral carotid PWV and E/A.[13] In 2012, Rabkin et al. examined the correlation between femoral heart PWV and left ventricular mass index in patients with controlled hypertension. A total of 20 patients with hypertension were controlled with an average age of $67.8+9.3$, and as many as $55 \%$ had received two or more types of antihypertensive drugs, femoral heart tests of PWV and echocardiography were performed. The results of this study are that there is a positive correlation between femoral heart PWV and LVMI. However, no significant correlation was found between the augmentation index and LVMI.[14]

The limitation of this study is that the number of samples is small so that the distribution of confounding factors cannot be removed entirely. No studies have been used to become a reference for the value of brachial-ankle PWV and left ventricular mass index in Indonesia.

\section{Conclusion}

There is a significant positive correlation between the brachial-ankle pulse wave velocity and the left ventricular mass index in hypertensive patients. Further research is needed with a larger sample number and a more extended research period to further strengthen the importance of the pulse wave examination to detect left ventricular hypertrophy in patients with hypertension.

Author Contributions: Conceptualization: D.F., B.S.P.; data collection: D.F., R.M. and E.P.B.M.; methodology: D.F. and B.S.P.; formal analysis: B.S.P. and E.P.B.M.; writing-original draft preparation: D.F., R.M. and E.P.B.M.; manuscript writing - review and editing: all authors; manuscript 
review: B.S.P. and E.P.B.M. All authors have read and agreed to the published version of the manuscript.

Funding: None.

Data Availability Statement: The data presented in this study are available on request from the corresponding author.

Acknowledgments: None

Conflicts of Interest: The authors declare there is no conflict of interest.

\section{References}

[1] Kearney PM, Whelton M, Reynolds K, Muntner P, Whelton PK, He J. Global burden of hypertension: analysis of worldwide data. Lancet 2005;365:217-23. https://doi.org/10.1016/S0140-6736(05)17741-1.

[2] Balitbangkes DR. Report on results of national basic health research (RISKESDAS) 2007. Jakarta: Depkes RI; 2008.

[3] Lavie CJ, Milani R V, Shah SB, Gilliland YE, Bernal JA, Dinshaw H, et al. Impact of left ventricular geometry on prognosis-a review of ochsner studies. Ochsner J 2008;8:11-7.

[4] Mackenzie IS, Wilkinson IB, Cockcroft JR. Assessment of arterial stiffness in clinical practice. QJM 2002;95:67-74. https://doi.org/10.1093/qjmed/95.2.67.

[5] Agabiti-Rosei E, Muiesan ML. Pathophysiology and treatment of hypertensive left ventricular hypertrophy. Dialogues Cardiovasc Med 2005;10:3-18.

[6] Munakata M. Utility of automated brachial ankle pulse wave velocity measurements in hypertensive patients. Am J Hypertens 2003;16:653-7. https://doi.org/10.1016/S0895-7061(03)00918-X.

[7] Díez J, Frohlich ED. A Translational Approach to Hypertensive Heart Disease. Hypertension 2010;55:1-8. https://doi.org/10.1161/HYPERTENSIONAHA.109.141887.

[8] Rosendorff C, Go O, Schmeidler J, Silverman JM, Beeri MS. Correlation of arterial blood pressure and compliance with left ventricular structure and function in the very elderly. J Am Soc Hypertens 2012;6:48-55. https://doi.org/10.1016/j.jash.2011.10.004.

[9] Aronow WS, Fleg JL, Pepine CJ, Artinian NT, Bakris G, Brown AS, et al. ACCF/AHA 2011 Expert Consensus Document on Hypertension in the Elderly. J Am Coll Cardiol 2011;57:2037-114. https://doi.org/10.1016/j.jacc.2011.01.008.

[10] Adji A, O’Rourke MF, Namasivayam M. Arterial Stiffness, Its Assessment, Prognostic Value, and Implications for Treatment. Am J Hypertens 2011;24:5-17. https://doi.org/10.1038/ajh.2010.192.

[11] Minesh R, Kunall G, Siva Y, Peter OJ. Relatioships of arterial and left ventricular stiffness indicators. J Clin Hypertens 2013;15:106-12.

[12] Schillaci G, Mannarino MR, Pucci G, Pirro M, Helou J, Savarese G, et al. Age-Specific Relationship of Aortic Pulse Wave Velocity With Left Ventricular Geometry and Function in Hypertension. Hypertension 2007;49:317-21. https://doi.org/10.1161/01.HYP.0000255790.98391.9b.

[13] Terentes-Printzios D, Vlachopoulos C, Bratsas A, Aznaouridis K, Alexopoulos N, Baou K, et al. Arterial stiffness is associated with left ventricular systolic and diastolic function in never-treated hypertensives. J Am Coll Cardiol 2010;55:A169.E1585. https://doi.org/10.1016/S0735-1097(10)61586-0.

[14] Rabkin SW, Chan SH. Correlation of pulse wave velocity with left ventricular mass in patients with hypertension once blood pressure has been normalized. Heart Int 2012;7:e5. https://doi.org/10.4081/hi.2012.e5. 